\title{
On a new semilocal convergence analysis for the Jarratt method
}

\author{
Ioannis K Argyros ${ }^{1}$, Yeol Je Cho ${ }^{2^{*}}$ and Sanjay Kumar Khattri ${ }^{3}$
}

\author{
"Correspondence: yjcho@gnu.ac.kr \\ ${ }^{2}$ Department of Mathematics \\ Education and the RINS, \\ Gyeongsang National University, \\ Jinju, 660-701, Korea \\ Full list of author information is \\ available at the end of the article
}

\begin{abstract}
We develop a new semilocal convergence analysis for the Jarratt method. Through our new idea of recurrent functions, we develop new sufficient convergence conditions and tighter error bounds. Numerical examples are also provided in this study.

MSC: 65H10;65G99;65J15; 47H17; 49M15

Keywords: Jarratt method; Newton-type methods; Banach space; Fréchet-derivative; majorizing sequence; recurrent functions
\end{abstract}

\section{Introduction}

In this study, we are concerned with the problem of approximating a locally unique solution $x^{\star}$ of the equation

$$
F(x)=0
$$

where $F$ is a Fréchet-differentiable operator defined on a convex subset $\mathcal{D}$ of a Banach space $\mathcal{X}$ with values in a Banach space $\mathcal{Y}$.

A large number of problems in applied mathematics and also in engineering are solved by finding the solutions of certain equations. For example, dynamic systems are mathematically modeled by difference or differential equations and their solutions usually represent the states of the systems. For the sake of simplicity, assume that a time-invariant system is driven by the equation $\dot{x}=Q(x)$ for some suitable operator $Q$, where $x$ is the state. Then the equilibrium states are determined by solving equation (1.1). Similar equations are used in the case of discrete systems. The unknowns of engineering equations can be functions (difference, differential and integral equations), vectors (systems of linear or nonlinear algebraic equations) or real or complex numbers (single algebraic equations with single unknowns). Except in special cases, the most commonly used solution methods are iterative - when starting from one or several initial approximations, a sequence is constructed that converges to a solution of the equation. Iteration methods are also applied for solving optimization problems. In such cases, the iteration sequences converge to an optimal solution of the problem at hand. Since all of these methods have the same recursive structure, they can be introduced and discussed in a general framework.

Many authors have developed high order methods for generating a sequence approximating $x^{\star}$. A survey of such results can be found in [1, and the references there] (see

\section{Springer}

(c) 2013 Argyros et al: licensee Springer. This is an Open Access article distributed under the terms of the Creative Commons Attribution License (http://creativecommons.org/licenses/by/2.0), which permits unrestricted use, distribution, and reproduction in any medium, provided the original work is properly cited. 
also [2-11]). The natural generalization of the Newton method is to apply a multipoint scheme. Suppose that we know the analytic expressions of $F\left(x_{n}\right), F^{\prime}\left(x_{n}\right)$ and $F^{\prime}\left(x_{n}\right)^{-1}$ at a recurrent step $x_{n}$ for each $n \geq 0$. In order to increase the order of convergence and to avoid the computation of the second Fréchet-derivative, we can add one more evaluation of $F\left(c_{1} x_{n}+c_{2} y_{n}\right)$ or $F^{\prime}\left(c_{1} x_{n}+c_{2} y_{n}\right)$, where $c_{1}$ and $c_{2}$ are real constants that are independent of $x_{n}$ and $y_{n}$, whereas $y_{n}$ is generated by a Newton-step. A two-point scheme for functions of one variable was found and developed by Ostrowski [11]. Following this idea, we provide a semilocal as well as a local convergence analysis for a fourth-order inverse free Jarratt-type method (JM) [1, 4] given by

$$
\begin{aligned}
& y_{n}=x_{n}-F^{\prime}\left(x_{n}\right)^{-1} F\left(x_{n}\right), \\
& \mathcal{B}_{n}=\mathcal{B}(n, F)=F^{\prime}\left(x_{n}\right)^{-1}\left(F^{\prime}\left(x_{n}+\frac{2}{3}\left(y_{n}-x_{n}\right)\right)-F^{\prime}\left(x_{n}\right)\right), \\
& x_{n+1}=y_{n}-\frac{3}{4} \mathcal{B}_{n}\left(\mathcal{I}-\frac{3}{2} \mathcal{B}_{n}\right)\left(y_{n}-x_{n}\right)
\end{aligned}
$$

for each $n \geq 0$. The fourth order of (JM) is the same as that of a two-step Newton method [1]. But the computational cost is less than that of Newton's method. In each step, we save one evaluation of the derivative and the computation of one inverse.

Here, we use our new idea of recurrent functions in order to provide new sufficient convergence conditions, which can be weaker than before [4]. Using this approach, the error bounds and the example on the distances are improved (see Example 3.5 and Remarks 3.6). This new idea can be used on other iterative methods [1].

\section{Semilocal convergence analysis of (JM)}

We present our Theorem 2.1 in [4] in an affine invariant form since $F^{\prime}\left(x_{0}\right)^{-1} F$ can be used for $F$ in the original proof of Theorem 2.1.

Theorem 2.1 Let $F: \mathcal{D} \subseteq \mathcal{X} \rightarrow \mathcal{Y}$ be thrice differentiable. Assume that there exist $x_{0} \in \mathcal{D}$, $L \geq 0, M \geq 0, N \geq 0$ and $\eta \geq 0$ such that

$$
\begin{aligned}
& F^{\prime}\left(x_{0}\right)^{-1} \in \mathcal{L}(\mathcal{Y}, \mathcal{X}), \\
& \left\|F^{\prime}\left(x_{0}\right)^{-1} F\left(x_{0}\right)\right\| \leq \eta, \\
& \left\|F^{\prime}\left(x_{0}\right)^{-1} F^{\prime \prime}(x)\right\| \leq M, \\
& \left\|F^{\prime}\left(x_{0}\right)^{-1} F^{\prime \prime \prime}(x)\right\| \leq N, \\
& \left\|F^{\prime}\left(x_{0}\right)^{-1}\left(F^{\prime \prime \prime}(x)-F^{\prime \prime \prime}(y)\right)\right\| \leq L\|x-y\|
\end{aligned}
$$

for each $x, y \in \mathcal{D}$,

$$
\begin{aligned}
& M\left(1+\frac{N}{6 M^{2}}+\frac{13 L}{36 M^{2}}\right)^{\frac{1}{3}} \leq K, \\
& h=K \eta \leq 0.46568
\end{aligned}
$$

and

$$
\bar{U}\left(x_{0}, v^{\star}\right)=\left\{x \in \mathcal{X},\left\|x-x_{0}\right\| \leq v^{\star}\right\} \subseteq \mathcal{D},
$$


where $v^{\star}$ and $v^{\star \star}$ are the zeros of functions

$$
g(t)=\frac{K}{2} t^{2}-t+\eta
$$

given by

$$
v^{\star}=\frac{1-\sqrt{1-2 h}}{h} \eta, \quad v^{\star \star}=\frac{1+\sqrt{1-2 h}}{h} \eta .
$$

Then the following hold:

(1) The scalar sequences $\left\{v_{n}\right\}$ and $\left\{w_{n}\right\}$ given by

$$
\left.\begin{array}{l}
w_{n}=v_{n}-g^{\prime}\left(v_{n}\right)^{-1} g\left(v_{n}\right), \\
b_{n}=g^{\prime}\left(v_{n}\right)^{-1}\left(g^{\prime}\left(v_{n}+\frac{2}{3}\left(w_{n}-v_{n}\right)\right)-g^{\prime}\left(v_{n}\right)\right), \\
v_{n+1}=w_{n}-\frac{3}{4} b_{n}\left(1-\frac{3}{2} b_{n}\right)\left(w_{n}-v_{n}\right)
\end{array}\right\}
$$

for each $n \geq 0$ are non-decreasing and converge to their common limit $v^{\star}$, so that

$$
v_{n} \leq w_{n} \leq v_{n+1} \leq w_{n+1}
$$

(2) The sequences $\left\{x_{n}\right\}$ and $\left\{y_{n}\right\}$ generated by $(J M)$ are well defined, remain in $\bar{U}\left(x_{0}, v^{\star}\right)$ for all $n \geq 0$ and converge to a unique solution $x^{\star} \in \bar{U}\left(x_{0}, v^{\star}\right)$ of the equation $F(x)=0$, which is the unique solution of the equation $F(x)=0$ in $U\left(x_{0}, v^{\star \star}\right)$. Moreover, the following estimates hold for all $n \geq 0$ :

$$
\begin{aligned}
& \left\|y_{n}-x_{n}\right\| \leq w_{n}-v_{n}, \\
& \left\|x_{n+1}-y_{n}\right\| \leq w_{n+1}-v_{n}, \\
& \left\|y_{n}-x^{\star}\right\| \leq v^{\star}-w_{n}, \\
& \left\|x_{n}-x^{\star}\right\| \leq v^{\star}-v_{n} \leq \frac{(1-\theta)^{2} \eta(\sqrt[3]{5} \theta)^{4^{n}-1}}{1-\frac{1}{\sqrt[3]{5}}(\sqrt[3]{5} \theta)^{4^{n}}},
\end{aligned}
$$

where

$$
\theta=\frac{v^{\star}}{v^{\star \star}}
$$

Remarks 2.2 The bounds of Theorem 2.1 can be improved under the same hypotheses and computational cost in two cases as follows.

Case 1. Define a function $g_{0}$ by

$$
g_{0}(t)=\frac{M_{0}}{2} t^{2}-t+\eta \text {. }
$$

In view of (2.2), there exists $M_{0} \in[0, M]$ such that

$$
\left\|F^{\prime}\left(x_{0}\right)^{-1}\left(F^{\prime}(x)-F^{\prime}\left(x_{0}\right)\right)\right\| \leq M_{0}\left\|x-x_{0}\right\|
$$


for all $x \in \mathcal{D}$. We can find upper bounds on the norms $\left\|F^{\prime}(x)^{-1} F^{\prime}\left(x_{0}\right)\right\|$ using $M_{0}$, which is actually needed, and not $K$ used in [4].

Note that

$$
M_{0} \leq K
$$

and $K / M_{0}$ can be arbitrarily large [1-3]. Using (2.19), it follows that, for any $x \in \bar{U}\left(x_{0}, v^{\star}\right)$,

$$
\left\|F^{\prime}\left(x_{0}\right)^{-1}\left(F^{\prime}(x)-F^{\prime}\left(x_{0}\right)\right)\right\| \leq M_{0}\left\|x-x_{0}\right\| \leq K\left\|x-x_{0}\right\| \leq K v^{\star}<1 .
$$

It follows from (2.21) and the Banach lemma on invertible operators [1] that $\left\|F^{\prime}(x)^{-1} F^{\prime}\left(x_{0}\right)\right\|$ exists and

$$
\left\|F^{\prime}(x)^{-1} F^{\prime}\left(x_{0}\right)\right\| \leq \frac{1}{1-M_{0}\left\|x-x_{0}\right\|} .
$$

We can use (2.21) instead of the less precise one used in [4]:

$$
\left\|F^{\prime}(x)^{-1} F^{\prime}\left(x_{0}\right)\right\| \leq \frac{1}{1-K\left\|x-x_{0}\right\|} .
$$

This suggests that more precise scalar majorizing sequences $\left\{\bar{v}_{n}\right\},\left\{\bar{w}_{n}\right\}$ can be used and they are defined as follows for initial iterates $\bar{v}_{0}=0, \bar{w}_{1}=\eta$ :

$$
\left.\begin{array}{l}
\bar{w}_{n}=\bar{v}_{n}-g_{0}^{\prime}\left(\bar{v}_{n}\right)^{-1} g\left(\bar{v}_{n}\right), \\
\bar{b}_{n}=b\left(n, g, g_{0}\right)=g_{0}^{\prime}\left(\bar{v}_{n}\right)^{-1}\left(g^{\prime}\left(\bar{v}_{n}+\frac{2}{3}\left(\bar{w}_{n}-\bar{v}_{n}\right)\right)-g^{\prime}\left(\bar{v}_{n}\right)\right), \\
\bar{v}_{n+1}=\bar{w}_{n}-\frac{3}{4} \bar{b}_{n}\left(1-\frac{3}{2} \bar{b}_{n}\right)\left(\bar{w}_{n}-\bar{v}_{n}\right) .
\end{array}\right\}
$$

A simple induction argument shows that, if $M_{0}<K$, then

$$
\begin{aligned}
& \bar{v}_{n}<v_{n}, \\
& \bar{w}_{n}<w_{n}, \\
& \bar{w}_{n}-\bar{v}_{n}<w_{n}-v_{n}, \\
& \bar{v}_{n+1}-\bar{w}_{n}<v_{n+1}-w_{n}
\end{aligned}
$$

and

$$
\bar{v}^{\star} \leq v^{\star}
$$

where

$$
\bar{v}^{\star}=\lim _{n \rightarrow \infty} \bar{v}_{n}
$$

Note also that if $M_{0}=K$, then $\bar{v}_{n}=v_{n}, \bar{w}_{n}=w_{n}$.

Case 2. In view of the upper bound for $\left\|F\left(x_{n+1}\right)\right\|$ obtained in Theorem 2.1 in [4] and (2.21), $\left\{t_{n}\right\},\left\{s_{n}\right\}$ given in (3.9) and (3.10) are also even more precise majorizing sequences 
for $\left\{x_{n}\right\}$ and $\left\{y_{n}\right\}$. Therefore, if they converge under certain conditions (see Lemma 3.2), then we can produce a new semilocal convergence theorem for (JM) with sufficient convergence conditions or bounds that can be better than the ones of Theorem 2.1 (see also Theorem 3.4 and Example 3.5).

Similar favorable comparisons (due to (2.20)) can be made with other iterative methods of the fourth order $[1,11]$.

\section{Semilocal convergence analysis of (JM) using recurrent functions}

We show the semilocal convergence of (JM) using recurrent functions. First, we need the following definition.

Definition 3.1 Let $L \geq 0, M_{0}>0, M>0, N \geq 0$ and $\eta>0$ be given constants. Define the polynomials on $[0,+\infty)$ for some $\alpha>0$ by

$$
\begin{aligned}
f_{1}(t)= & (1+M \eta) M \eta t+4 M_{0} \alpha(\alpha+2) \eta-2 \alpha, \\
g(t)= & \left.M(1+M \eta) t^{2}+\left[4 M_{0} \alpha(1+\alpha)\right]-M(1+M \eta)\right] t-4 M_{0} \alpha, \\
h_{1}(t)= & M_{0} \eta(1+\alpha) t^{2}+M_{0} \eta(1+\alpha) t+\frac{M \alpha^{2} \eta}{2}+\frac{13 L \eta^{3}}{108} \\
& +\frac{2 N \alpha \eta}{9 M}+\frac{2 \alpha M \eta}{3}-1, \\
g_{1}(t)= & M_{0} \eta(1+\alpha) t^{2}+\left(\frac{M \alpha^{2}}{2}+\frac{13 L \eta^{2}}{108}+\frac{2 N \alpha}{9 M}+\frac{2 \alpha M}{3}-M_{0} \eta\right) t \\
& -\left(\frac{M \alpha^{2}}{2}+\frac{13 L \eta^{2}}{108}+\frac{2 N \alpha}{9 M}+\frac{2 \alpha M}{3}\right) .
\end{aligned}
$$

Moreover, define a scalar $\phi_{0}$ by

$$
\phi_{0}=\frac{\left[\frac{M \alpha^{2}}{2}+\frac{13 L \eta^{3}}{108}+\frac{2 N \alpha \eta}{9 M}+\frac{2 \alpha M \eta}{3}\right]}{1-M_{0}\left[\eta+\frac{M(1+M \eta)}{2} \eta^{2}\right]} .
$$

The polynomials $f_{1}, g, g_{1}$ have unique positive roots denoted by $\phi_{f_{1}}, \phi_{g}$ and $\phi_{g_{1}}$ (given in an explicit form), respectively, by the Descartes rule of signs. Moreover, assume

$$
M_{0}\left[\eta+\frac{M(1+M \eta)}{2} \eta^{2}\right]<1
$$

and

$$
\frac{M \alpha^{2} \eta}{2}+\frac{13 L \eta^{3}}{108}+\frac{2 N \alpha \eta}{9 M}+\frac{2 \alpha M \eta}{3}<1 .
$$

Under the conditions (3.1), (3.2), respectively,

$$
\phi_{0}>0
$$

and the polynomial $h_{1}$ has a unique positive root $\phi_{h_{1}}$. 
Set $\phi_{1}=\min \left\{\phi_{h_{1}}, \phi_{f_{1}}, \phi_{g}, \phi_{g_{1}}, 1\right\}$. Furthermore, assume

$$
\phi_{0} \leq \phi_{1}
$$

If $\phi_{1}=1$, then assume that (3.3) holds as a strict inequality. From now on (3.1)-(3.3) constitute the $(C)$ conditions.

We can show the following result on the majorizing sequences for (JM).

Lemma 3.2 Under the $(\mathrm{C})$ conditions, choose

$$
\phi \in\left[\phi_{0}, \phi_{1}\right] \quad \text { if } \phi_{1} \neq 1 \text { and } \phi \in\left[\phi_{0}, 1\right) \text { if } \phi_{1}=1 \text {. }
$$

Then the scalar sequences $\left\{s_{n}\right\},\left\{t_{n}\right\}$ given by

$$
\begin{aligned}
t_{0}=0, \quad s_{0}=\eta & \\
t_{n+1}= & s_{n}+\frac{M\left(1+M\left(s_{n}-t_{n}\right)\right)\left(s_{n}-t_{n}\right)^{2}}{2\left(1-M_{0} t_{n}\right)^{2}}, \\
s_{n+1}= & t_{n+1}+\frac{1}{1-M_{0} t_{n+1}}\left[\frac{M\left(t_{n+1}-s_{n}\right)^{2}}{2}+\frac{13 L\left(s_{n}-t_{n}\right)^{4}}{108}\right. \\
& \left.+\frac{N M\left(s_{n}-t_{n}\right)^{4}}{9\left(1-M_{0} t_{n}\right)}+\frac{M^{3}\left(s_{n}-t_{n}\right)^{4}}{3\left(1-M_{0} t_{n}\right)^{2}}\right]
\end{aligned}
$$

are non-decreasing, bounded from above by

$$
t^{\star \star}=\left(1+\frac{\alpha}{1-\phi}\right) \eta
$$

and converge to their unique least upper bound $t^{\star} \in\left[0, t^{\star \star}\right]$. Moreover, the following estimate holds:

$$
0 \leq s_{n+1}-t_{n+1} \leq \phi\left(s_{n}-t_{n}\right),
$$

where

$$
\alpha=\frac{M(1+M \eta) \eta}{2} .
$$

Proof We show, using induction on $k$, that

$$
0 \leq \frac{M\left(1+M\left(s_{k}-t_{k}\right)\right)\left(s_{k}-t_{k}\right)}{2\left(1-M_{0} t_{k}\right)^{2}} \leq \alpha
$$

and

$$
\begin{aligned}
0 & \leq \frac{1}{1-M_{0} t_{n+1}}\left[\frac{M \alpha^{2}}{2}\left(s_{k}-t_{k}\right)+\frac{13 L}{108}\left(s_{k}-t_{k}\right)^{3}+\frac{N M\left(s_{k}-t_{k}\right)^{3}}{9\left(1-M_{0} t_{k}\right)}+\frac{M^{3}\left(s_{k}-t_{k}\right)^{3}}{3\left(1-M_{0} t_{k}\right)^{2}}\right] \\
& \leq \phi
\end{aligned}
$$


The estimate (3.8) holds for $k=0$ by the choice of $\alpha$. Moreover, the estimates (3.7) and (3.9) hold for $n=0$ by (3.5), the choice of $\phi_{0}$ and (3.4). Let us assume (3.7)-(3.9) hold for all $k \leq n$. We have in turn by the induction hypotheses:

$$
\begin{aligned}
& s_{k}- t_{k} \leq \phi\left(s_{k-1}-t_{k-1}\right) \leq \cdots \leq \phi^{k}\left(s_{0}-t_{0}\right)=\phi^{k} \eta, \\
& t_{k+1} \leq s_{k}+\alpha\left(s_{k}-t_{k}\right) \leq t_{k}+\alpha \phi^{k} \eta+\phi^{k} \eta \\
& \leq s_{k-1}+\alpha\left(s_{k-1}-t_{k-1}\right)+\alpha \phi^{k} \eta+\phi^{k} \eta \\
& \leq s_{k-1}+\alpha \phi^{k-1} \eta+\alpha \phi^{k} \eta+\phi^{k} \eta \\
& \leq \cdots \\
& \leq s_{0}+\alpha \eta\left(1+\cdots+\phi^{k}\right)+\phi^{k} \eta<\eta+\frac{\alpha \eta\left(1-\phi^{k+1}\right)}{1-\phi}+\phi^{k} \eta, \\
& \frac{M\left(s_{k}-t_{k}\right)}{2\left(1-M_{0} t_{k}\right)^{2}}+\frac{M^{2}\left(s_{k}-t_{k}\right)^{2}}{2\left(1-M_{0} t_{k}\right)^{2}} \leq \alpha
\end{aligned}
$$

or

$$
\frac{2}{M^{2}} \frac{M}{2} \frac{\left(s_{k}-t_{k}\right)}{\left(1-M_{0} t_{k}\right)^{2}}+\frac{\left(s_{k}-t_{k}\right)^{2}}{\left(1-M_{0} t_{k}\right)^{2}} \leq \frac{2 \alpha}{M^{2}}
$$

or

$$
\left(\frac{s_{k}-t_{k}}{1-M_{0} t_{k}}\right)^{2} \leq \frac{2 \alpha}{M^{2}}
$$

and

$$
\begin{aligned}
\frac{N M\left(s_{k}-t_{k}\right)^{3}}{9\left(1-M_{0} t_{k}\right)} & =\frac{N M\left(1-M_{0} t_{k}\right)\left(s_{k}-t_{k}\right)^{3}}{9\left(1-M_{0} t_{k}\right)^{2}} \\
& =\frac{N M}{9}\left(1-M_{0} t_{k}\right)\left(\frac{s_{k}-t_{k}}{1-M_{0} t_{k}}\right)^{2}\left(s_{k}-t_{k}\right) \\
& \leq \frac{2}{M^{2}} \frac{N M}{9}\left(s_{k}-t_{k}\right) \alpha=\frac{2 N \alpha}{9 M}\left(s_{k}-t_{k}\right), \\
\frac{M^{3}\left(s_{k}-t_{k}\right)^{3}}{3\left(1-M_{0} t_{k}\right)^{2}} & =\frac{M^{3}\left(s_{k}-t_{k}\right)}{3} \frac{\left(s_{k}-t_{k}\right)^{2}}{\left(1-M_{0} t_{k}\right)^{2}} \\
& \leq \frac{M^{3}}{3}\left(s_{k}-t_{k}\right) \frac{2 \alpha}{M^{2}}=\frac{2 M \alpha}{3}\left(s_{k}-t_{k}\right) .
\end{aligned}
$$

Hence, instead of (3.9), we can show

$$
\begin{aligned}
0 & \leq \frac{1}{\left(1-M_{0} t_{k+1}\right)}\left[\frac{M \alpha^{2}}{2}\left(s_{k}-t_{k}\right)+\frac{13 L}{108}\left(s_{k}-t_{k}\right)^{3}+\frac{2 N \alpha}{9 M}\left(s_{k}-t_{k}\right)+\frac{2 M \alpha\left(s_{k}-t_{k}\right)}{3}\right] \\
& \leq \phi
\end{aligned}
$$

The estimate (3.8) can be written as

$$
M\left(1+M \phi^{k} \eta\right) \phi^{k} \eta \leq 2 \alpha\left(1-M_{0} t_{k}\right)^{2}
$$


or

$$
M\left(1+M \phi^{k} \eta\right) \phi^{k} \eta \leq 2 \alpha+2 \alpha M_{0}^{2} t_{k}^{2}-4 M_{0} \alpha t_{k}
$$

So, we can show, instead of (3.8),

$$
M\left(1+M \phi^{k} \eta\right) \phi^{k} \eta+4 M_{0} \alpha t_{k} \leq 2 \alpha
$$

or

$$
M\left(1+M \phi^{k} \eta\right) \phi^{k} \eta+4 M_{0} \alpha\left[\eta+\alpha \eta\left(\frac{1-\phi^{k}}{1-\phi}\right)+\phi^{k-1} \eta\right]-2 \alpha \leq 0
$$

The estimate (3.11) motivates us to define polynomials $\bar{f}_{k}$ on $[0,1)($ for $\phi=t)$ by

$$
\begin{aligned}
\bar{f}_{k}(t) & =M\left(1+M \phi^{k} \eta\right) t^{k} \eta+4 M_{0} \alpha \eta\left[1+\alpha\left(\frac{1-t^{k}}{1-t}\right)+t^{k-1}\right]-2 \alpha \\
& =M t^{k} \eta+M^{2} \eta^{2} t^{2 k}+4 M_{0} \alpha\left[1+\alpha\left(\frac{1-t^{k}}{1-t}\right)+t^{k-1}\right] \eta-2 \alpha
\end{aligned}
$$

or, since $t^{2} \leq t$ for $t \in[0,1]$, define the polynomials $f_{k}$ on $[0,1)$ by

$$
f_{k}(t)=M t^{k} \eta+M^{2} \eta^{2} t^{k}+4 M_{0} \alpha\left[1+\alpha\left(\frac{1-t^{k}}{1-t}\right)+t^{k-1}\right] \eta-2 \alpha .
$$

We need a relationship between two consecutive polynomials $f_{k}$ :

$$
\begin{aligned}
f_{k+1}(t) & =M t^{k+1} \eta+M^{2} \eta^{2} t^{k+1}+4 M_{0} \alpha\left[1+\alpha\left(\frac{1-t^{k+1}}{1-t}\right)+t^{k}\right] \eta-2 \alpha+f_{k}(t)-f_{k}(t) \\
& =f_{k}(t)+g(t) t^{k-1} \eta
\end{aligned}
$$

where $g$ and its unique positive root $\phi_{g} \in[0,1)$ are given in Definition 3.1. The estimate (3.11) is true if

$$
f_{k}(\phi) \leq 0
$$

or, if

$$
f_{1}(\phi) \leq 0
$$

since by (3.14) we have

$$
f_{k}(\phi)=f_{1}(\phi)
$$

But (3.16) is true by the definition of $\phi_{f_{1}}$ and (3.4). Define

$$
f_{\infty}(\phi)=\lim _{k \rightarrow \infty} f_{k}(\phi)
$$


Then we also have

$$
f_{\infty}(\phi)=\lim _{k \rightarrow \infty} f_{k}(\phi)=\lim _{k \rightarrow \infty} f_{1}(\phi) \leq \lim _{k \rightarrow \infty} 0=0 .
$$

This completes the induction for (3.8). The estimate (3.10) is true if

$$
\frac{M \alpha^{2} \phi^{k} \eta}{2}+\frac{13 L}{108}\left(\phi^{k} \eta\right)^{3}+\frac{2 N \alpha}{9 M} \phi^{k} \eta+\frac{2 \alpha M}{3} \phi^{k} \eta \leq \phi\left(1-M_{0} t_{k+1}\right)
$$

or

$$
\begin{gathered}
\frac{M \alpha^{2} \phi^{k} \eta}{2}+\frac{13 L}{108}\left(\phi^{k} \eta\right)^{3}+\frac{2 N \alpha}{9 M} \phi^{k} \eta+\frac{2 \alpha M}{3} \phi^{k} \\
+\phi M_{0}\left[1+\alpha\left(\frac{1-\phi^{k+1}}{1-\phi}\right)+\phi^{k}\right] \eta-\phi \leq 0 .
\end{gathered}
$$

The estimate (3.20) motivates us to define polynomials $h_{k}$ on $[0,1)$ by

$$
\begin{aligned}
h_{k}(t)= & \frac{M \alpha^{2}}{2} t^{k} \eta+\frac{13 L}{108} \eta^{3} t^{k}+\frac{2 N \alpha}{9 M} t^{k} \eta+\frac{2 \alpha M}{3} t^{k} \eta \\
& +\phi M_{0}\left[1+\alpha\left(\frac{1-t^{k+1}}{1-t}\right)+t^{k}\right] \eta-\phi .
\end{aligned}
$$

We need a relationship between two consecutive polynomials $h_{k}$ :

$$
\begin{aligned}
h_{k+1}(t)= & \frac{M \alpha^{2}}{2} t^{k+1} \eta+\frac{13 L}{108} \eta^{3} t^{k+1}+\frac{2 N \alpha}{9 M} t^{k+1} \eta+\frac{2 \alpha M}{3} t^{k+1} \eta \\
& +\phi M_{0}\left[1+\alpha\left(\frac{1-t^{k+2}}{1-t}\right)+t^{k+1}\right] \eta-\phi-\frac{M \alpha^{2}}{2} t^{k} \eta-\frac{13 L}{108} \eta^{3} t^{k}-\frac{2 \alpha M}{3} t^{k} \eta \\
& -\frac{2 N \alpha}{9 M} t^{k} \eta-\phi M_{0}\left[1+\alpha\left(\frac{1-t^{k+1}}{1-t}\right)+t^{k}\right]+\phi+h_{k}(t)
\end{aligned}
$$

and so

$$
f_{k+1}(t)=h_{k}(t)+g_{1}(t) t^{k} \eta
$$

where $g_{1}$ and the unique positive root $\phi_{g_{1}}$ are given in Definition 3.1. The estimate (3.20) is true if

$$
h_{k}(\phi) \leq 0
$$

or, if

$$
h_{1}(\phi) \leq 0
$$

since

$$
h_{k}(\phi)=h_{1}(\phi) .
$$


But (3.24) is true by the definition of $\phi_{h_{1}}$ and (3.4). Define a function $h_{\infty}$ on $[0,1)$ by

$$
h_{\infty}(\phi)=\lim _{k \rightarrow \infty} h_{k}(\phi)
$$

Then we have

$$
h_{\infty}(\phi)=\lim _{k \rightarrow \infty} h_{k}(\phi)=\lim _{k \rightarrow \infty} h_{1}(\phi) \leq \lim _{k \rightarrow \infty} 0=0 .
$$

This completes the induction for (3.4)-(3.9). It follows that the sequences $\left\{s_{n}\right\}$ and $\left\{t_{n}\right\}$ are non-decreasing, bounded from above by $t^{\star \star}$ given in a closed form by (3.6) and converge to their unique least upper bound $t^{\star} \in\left[0, t^{\star \star}\right]$. This completes the proof.

Proposition $3.3[1,4]$ Under the hypotheses of Lemma 3.2, further assume

$$
\sqrt[3]{b} \eta<1
$$

where

$$
b=a+\frac{M^{3}}{8} \quad \text { and } \quad a=\frac{M^{3}}{3}+\frac{N M}{q}+\frac{13 L}{108} .
$$

Fix

$$
q \in\left(\sqrt[3]{b}, \frac{1}{\eta}\right), \quad \eta \neq 0
$$

Define the parameters $p_{0}, p$ by

$$
\left.\begin{array}{l}
p_{0}=\frac{1}{M_{0}}\left(1-\frac{\sqrt[3]{b}}{q}\right), \\
p=\frac{M q}{2 \sqrt[3]{b}}, \quad b \neq 0,
\end{array}\right\}
$$

and a function $g_{3}$ on $[1,1 / q)$ by

$$
g_{3}(t)=t+\frac{1}{q}+\frac{p}{q^{2}}\left(\frac{(q t)^{2}}{1-(q t)^{2}}+t^{2}\right) .
$$

Moreover, assume

$$
\min \left\{t_{1}, g_{3}(\eta)\right\} \leq p_{0}
$$

Then the following estimates hold for all $k \geq 0$ :

$$
\left.\begin{array}{l}
t_{k+1}-s_{k} \leq \frac{p}{q^{2}} \sqrt{(q \eta)^{4^{k+1}}} \\
s_{k}-t_{k} \leq \frac{1}{q}(q \eta)^{4^{k}}
\end{array}\right\}
$$

Proof We show

$$
s_{m+1}-t_{m+1} \leq q^{3}\left(s_{m}-t_{m}\right)^{4} .
$$


If the estimate (3.34) holds, then we have

$$
\begin{aligned}
q\left(s_{m+1}-t_{m+1}\right) & \leq\left(q\left(s_{m}-t_{m}\right)\right)^{4^{1}} \\
& \leq(q \eta)^{4^{m+1}}
\end{aligned}
$$

which implies the second equation in (3.33). We have the estimate

$$
\begin{aligned}
& \frac{M}{2}\left(t_{m+1}-s_{m}\right)^{2}+\frac{13 L}{108}\left(s_{m}-t_{m}\right)^{4}+\frac{N M}{9\left(1-M_{0} t_{m}\right)}\left(s_{m}-t_{m}\right)^{4}+\frac{M^{3}}{3\left(1-M_{0} t_{m}\right)^{2}}\left(s_{m}-t_{m}\right)^{4} \\
& \leq \frac{M}{2}\left(\frac{M\left(s_{m}-t_{m}\right)^{2}}{2\left(1-M_{0} t_{m}\right)}\right)^{2}+\frac{13 L}{108}\left(s_{m}-t_{m}\right)^{4} \\
& \quad+\frac{N M}{9\left(1-M_{0} t_{m}\right)}\left(s_{m}-t_{m}\right)^{4}+\frac{M^{3}}{3\left(1-M_{0} t_{m}\right)^{2}}\left(s_{m}-t_{m}\right)^{4} \\
& \leq \frac{M^{3}}{8} \frac{\left(s_{m}-t_{m}\right)^{4}}{\left(1-M_{0} t_{m}\right)^{2}}+\frac{13 L}{108} \frac{\left(s_{m}-t_{m}\right)^{4}}{\left(1-M_{0} t_{m}\right)^{2}}\left(1-M_{0} t_{m}\right)^{2} \\
& \quad+\frac{N M\left(s_{m}-t_{m}\right)^{4}}{9\left(1-M_{0} t_{m}\right)^{2}}\left(1-M_{0} t_{m}\right)+\frac{M^{3}}{3} \frac{\left(s_{m}-t_{m}\right)^{4}}{\left(1-M_{0} t_{m}\right)^{2}} \\
& \leq \frac{b\left(s_{m}-t_{m}\right)^{4}}{\left(1-M_{0} t_{m}\right)^{2}}
\end{aligned}
$$

that is, we have

$$
s_{m+1}-t_{m+1} \leq \frac{b\left(s_{m}-t_{m}\right)^{4}}{\left(1-M_{0} t_{m+1}\right)\left(1-M_{0} t_{m}\right)}
$$

Instead of showing (3.34), we can show

$$
\frac{b\left(s_{m}-t_{m}\right)^{4}}{\left(1-M_{0} t_{m}\right)^{2}\left(1-M_{0} t_{m+1}\right)} \leq q^{3}\left(s_{m}-t_{m}\right)^{4}
$$

or

$$
\frac{b}{\left(1-M_{0} t_{m+1}\right)^{3}} \leq q^{3},
$$

or

$$
t_{m+1} \leq p_{0} .
$$

By the hypothesis (3.32), we have

$$
t_{1} \leq p_{0} .
$$

Assume

$$
t_{m} \leq p_{0} .
$$


We also have

$$
\begin{aligned}
t_{m+1}-s_{m} & =\frac{M\left(s_{m}-t_{m}\right)^{2}}{2\left(1-M_{0} t_{m}\right)} \\
& \leq \frac{M q}{2 \sqrt[3]{b}}\left(s_{m}-t_{m}\right)^{2}=p\left(s_{m}-t_{m}\right)^{2} .
\end{aligned}
$$

We get in turn

$$
\begin{aligned}
t_{m+1} & \leq\left(s_{m}-t_{m}\right)+\left(t_{m}-s_{m-1}\right)+\cdots+\left(t_{1}-s_{0}\right)+s_{0}+p\left(s_{m}-t_{m}\right)^{2} \\
& \leq \eta+\frac{1}{q}(q \eta)^{4^{m}}+p\left(\left(s_{m}-t_{m}\right)^{2}+\left(s_{m-1}-t_{m-1}\right)^{2}+\cdots+\left(s_{0}-t_{0}\right)^{2}\right) \\
& \leq \eta+\frac{1}{q}(q \eta)^{4^{m}}+\frac{p}{q^{2}}\left((q \eta)^{\left(4^{m}\right)^{2}}+(q \eta)^{\left(4^{m-1}\right)^{2}}+\cdots+\eta^{2}\right) \\
& =\eta+\frac{1}{q}(q \eta)^{4^{m}}+\frac{p}{q^{2}}\left(\left((q \eta)^{\frac{1}{2}}\right)^{4^{m+1}}+\left((q \eta)^{\frac{1}{2}}\right)^{4^{m}}+\cdots+\left((q \eta)^{\frac{1}{2}}\right)^{4^{1}}+\eta^{2}\right) \\
& \leq \eta+\frac{1}{q}+\frac{p}{q^{2}}\left((q \eta)^{2(m+1)}+(q \eta)^{2 m}+\cdots+(q \eta)^{2}+\eta^{2}\right) \\
& \leq \eta+\frac{1}{q}\left(\frac{(q \eta)^{2}}{1-(q \eta)^{2}}+\eta^{2}\right)=g_{3}(\eta) \leq p_{0},
\end{aligned}
$$

which completes the induction for (3.38). This completes the proof.

Theorem 3.4 Under the hypotheses (3.1)-(3.5) and (3.23), further assume that the hypotheses of Lemma 3.2 hold and

$$
\bar{U}\left(x, t^{\star}\right) \subseteq D
$$

Then the sequences $\left\{x_{n}\right\}$ and $\left\{y_{n}\right\}$ generated by (JM) are well defined, remain in $\bar{U}\left(x, t^{\star}\right)$ for all $n \geq 0$ and converge to a unique solution $x^{\star}$ of the equation $F(x)=0$ in $\bar{U}\left(x, t^{\star}\right)$. Moreover, the following estimates hold:

$$
\begin{aligned}
& \left\|y_{n}-x_{n}\right\| \leq s_{n}-t_{n}, \\
& \left\|x_{n+1}-y_{n}\right\| \leq t_{n+1}-s_{n}, \\
& \left\|x_{n}-x^{\star}\right\| \leq t^{\star}-t_{n}, \\
& \left\|y_{n}-x^{\star}\right\| \leq t^{\star}-s_{n} .
\end{aligned}
$$

Furthermore, under the hypotheses of Proposition 3.3, the estimates (3.33) also hold. Finally, if $R \geq t^{\star}$ such that

$$
U(x, R) \subseteq \mathcal{D}
$$

and

$$
R \leq \frac{2}{M_{0}}-t^{\star}
$$

then the solution $x^{\star}$ is unique in $U(x, R)$. 
Example 3.5 Let $\mathcal{X}=\mathcal{Y}=\mathbb{R}^{2}, \mathcal{D}=[1,3]^{2}, x_{0}=(2,2)^{T}$ and define a function $F$ on $\mathcal{D}$ by

$$
F(x)=\left(\xi_{1}^{3}-2 \xi_{1}-5, \xi_{2}^{3}-2 \xi_{2}-5\right)^{T}
$$

Using (2.1)-(2.7), we obtain

$$
\eta=0.1, \quad M=1.8, \quad N=0.6, \quad M_{0}=1.5, \quad L=0, \quad K=1.8183312
$$

and

$$
h=0.18183312<0.46568 .
$$

Hence the conclusions of Theorem 2.1 hold for the equation $F(x)=0$. Considering the hypotheses of Theorem 3.4, from Lemma 3.2, we have

$$
\alpha=0.1062000000
$$

and, from Definition 3.1, we get

$$
\begin{aligned}
& \phi_{f_{1}}=0.3681400000, \quad \phi_{g}=0.9756327521, \\
& \phi_{h_{1}}=1.987765245, \quad \phi_{g_{1}}=0.9500677043 .
\end{aligned}
$$

Consequently, from the definition of $\phi_{1}$ (see Definition 3.1), we obtain

$$
\phi_{1}=\phi_{f_{1}}=0.3681400000 \text {, }
$$

and from the definition of $\phi_{0}$ (see Definition 3.1), we obtain

$$
\phi_{0}=0.02839241631
$$

We see the assumption $\phi_{0}<\phi_{1}$ (see equation (3.3) in Definition 3.1) is also valid. Furthermore, from the equation (3.4) (see Lemma 3.2), we consider

$$
\phi=0.2 \text {. }
$$

From the equation (3.6),

$$
t^{\star \star}=0.1265500000
$$

Hence the conditions of Theorem 3.4 are also satisfied. Additionally, to verify the claims about the sequences $\left\{s_{n}\right\}$ and $\left\{t_{n}\right\}$ (see equation (3.5)), we produce Table 1 .

From Table 1, we observe the following:

- The sequences $\left\{t_{n}\right\}$ and $\left\{s_{n}\right\}$ are non-decreasing.

The sequences $\left\{t_{n}\right\}$ and $\left\{s_{n}\right\}$ are bounded from above by $t^{\star \star}$.

The estimate (3.7) holds. 
Table 1 The scalar sequences $\left\{s_{n}\right\}$ and $\left\{t_{n}\right\}$ are given by equation (3.5) in Lemma 3.2

\begin{tabular}{lllll}
\hline $\boldsymbol{n}$ & $\boldsymbol{t}_{\boldsymbol{n}}$ & $\boldsymbol{s}_{\boldsymbol{n}}$ & $\boldsymbol{s}_{\boldsymbol{n}}-\boldsymbol{t}_{\boldsymbol{n}}$ & $\boldsymbol{\phi}\left(\boldsymbol{s}_{\boldsymbol{n}}-\boldsymbol{t}_{\boldsymbol{n}}\right)$ \\
\hline 0 & $0.000000 \times 10^{+00}$ & $1.000000 \times 10^{-01}$ & $1.000000 \times 10^{-01}$ & $2.000000 \times 10^{-02}$ \\
1 & $1.106200 \times 10^{-01}$ & $1.109892 \times 10^{-01}$ & $3.691608 \times 10^{-04}$ & $7.383216 \times 10^{-05}$ \\
2 & $1.109893 \times 10^{-01}$ & $1.109893 \times 10^{-01}$ & $9.907814 \times 10^{-14}$ & $1.981563 \times 10^{-14}$ \\
3 & $1.109893 \times 10^{-01}$ & $1.109893 \times 10^{-01}$ & $5.147480 \times 10^{-52}$ & $1.029496 \times 10^{-52}$ \\
4 & $1.109893 \times 10^{-01}$ & $1.109893 \times 10^{-01}$ & $3.750264 \times 10^{-205}$ & $7.500528 \times 10^{-206}$ \\
5 & $1.109893 \times 10^{-01}$ & $1.109893 \times 10^{-01}$ & $1.056649 \times 10^{-817}$ & $2.113298 \times 10^{-818}$ \\
6 & $1.109893 \times 10^{-01}$ & $1.109893 \times 10^{-01}$ & $0.000000 \times 10^{+00}$ & $0.000000 \times 10^{+00}$ \\
\hline
\end{tabular}

Table 2 The scalar sequences $\left\{w_{n}\right\}$ and $\left\{v_{n}\right\}$ are given by equation (2.11) in Theorem 2.1

\begin{tabular}{llll}
\hline $\boldsymbol{n}$ & $\boldsymbol{w}_{\boldsymbol{n}}$ & $\boldsymbol{v}_{\boldsymbol{n}}$ & $\boldsymbol{w}_{\boldsymbol{n}}-\boldsymbol{v}_{\boldsymbol{n}}$ \\
\hline 0 & $1.000000 \times 10^{-01}$ & $0.000000 \times 10^{+00}$ & $1.000000 \times 10^{-01}$ \\
1 & $1.112526 \times 10^{-01}$ & $1.107448 \times 10^{-01}$ & $5.078254 \times 10^{-04}$ \\
2 & $1.112529 \times 10^{-01}$ & $1.112529 \times 10^{-01}$ & $4.913882 \times 10^{-13}$ \\
3 & $1.112529 \times 10^{-01}$ & $1.112529 \times 10^{-01}$ & $1.527672 \times 10^{-41}$ \\
4 & $1.112529 \times 10^{-01}$ & $1.112529 \times 10^{-01}$ & $1.476527 \times 10^{-98}$ \\
5 & $1.112529 \times 10^{-01}$ & $1.112529 \times 10^{-01}$ & $1.379314 \times 10^{-212}$ \\
6 & $1.112529 \times 10^{-01}$ & $1.112529 \times 10^{-01}$ & $1.203670 \times 10^{-440}$ \\
7 & $1.112529 \times 10^{-01}$ & $1.112529 \times 10^{-01}$ & $9.166325 \times 10^{-897}$ \\
8 & $1.112529 \times 10^{-01}$ & $1.112529 \times 10^{-01}$ & $5.315832 \times 10^{-1809}$ \\
9 & $1.112529 \times 10^{-01}$ & $1.112529 \times 10^{-01}$ & $0.000000 \times 10^{+00}$ \\
\hline
\end{tabular}

Let us now compare the bounds between Theorems 2.1 and 3.4. From equation (2.10), we get

$$
v^{\star}=1.112529 \times 10^{-01} \text { and } v^{\star \star}=9.886567 \times 10^{-01} .
$$

From the equation (2.11), for $v[0]=0$, we obtain Table 2 .

Comparing Tables 1 and 2, we observe that the bounds of Theorem 3.4 are finer than those of Theorem 2.1. That is, $s_{n}-t_{n} \leq w_{n}-v_{n}$ for all $n=0,1,2, \ldots$ Considering the hypotheses of Proposition 3.3, we have for $q=4$

$$
\begin{aligned}
& a=2.214000000, \quad \sqrt[3]{b}=1.433056854, \quad p_{0}=0.4278238577 \\
& p=2.512112474 \quad \text { and } \quad g_{3}(\eta)=0.3814761712<p_{0} .
\end{aligned}
$$

From Table 1 and the preceding data, we note that $\min \left\{t_{1}, g_{3}(\eta)\right\}<p_{0}$. Consequently, the assumption (3.32) is true. Additionally, to ascertain the estimate (3.33), we form Table 3.

In Table 3, we observe that the estimates (3.33) are also true. Hence the conclusions of Proposition 3.3 also hold for the equation $F(x)=0$.

Remarks 3.6 [1, 6, 7] (1) The condition (3.32) can be replaced by a stronger, but easier to check

$$
\frac{2 \eta}{2-\delta} \leq p_{0}
$$

for $\delta \in I$ (see (3.13) and (3.21)).

The best possible choice for $\delta$ seems to be $\delta=\delta_{3}$. Let

$$
\delta_{3}=\max \left\{2 \delta_{1}, 2 \delta_{2}, \delta_{0}\right\}
$$


Table 3 To validate the estimate (3.33) of Proposition 3.3

\begin{tabular}{rllll}
\hline $\boldsymbol{n}$ & $\boldsymbol{s}_{\boldsymbol{n}}-\boldsymbol{t}_{\boldsymbol{n}}$ & $\left(\boldsymbol{t}_{\boldsymbol{n + 1}}-\boldsymbol{s}_{\boldsymbol{n}}\right)$ & $\frac{\boldsymbol{p}}{\boldsymbol{q}^{\mathbf{2}}} \sqrt{(\boldsymbol{q} \boldsymbol{\eta})^{\mathbf{4}^{\boldsymbol{k}+\mathbf{1}}}}$ & $\mathbf{1}_{\boldsymbol{q}}(\boldsymbol{q} \boldsymbol{\eta})^{\mathbf{4}^{\boldsymbol{k}}}$ \\
\hline 0 & $1.000000 \times 10^{-01}$ & $1.062000 \times 10^{-02}$ & $2.512112 \times 10^{-02}$ & $1.000000 \times 10^{-01}$ \\
1 & $3.691608 \times 10^{-04}$ & $1.764238 \times 10^{-07}$ & $1.028961 \times 10^{-04}$ & $6.400000 \times 10^{-03}$ \\
2 & $9.907814 \times 10^{-14}$ & $1.271658 \times 10^{-26}$ & $2.896268 \times 10^{-14}$ & $1.073742 \times 10^{-07}$ \\
3 & $5.147480 \times 10^{-52}$ & $3.432448 \times 10^{-103}$ & $1.818017 \times 10^{-52}$ & $8.507059 \times 10^{-27}$ \\
4 & $3.750264 \times 10^{-205}$ & $1.821958 \times 10^{-409}$ & $2.822505 \times 10^{-205}$ & $3.351952 \times 10^{-103}$ \\
5 & $1.056649 \times 10^{-817}$ & $1.446359 \times 10^{-1634}$ & $1.639764 \times 10^{-816}$ & $8.079252 \times 10^{-409}$ \\
6 & $0.000000 \times 10^{+00}$ & $0.000000 \times 10^{+00}$ & $1.867962 \times 10^{-3261}$ & $2.726870 \times 10^{-1631}$ \\
7 & $0.000000 \times 10^{+00}$ & $0.000000 \times 10^{+00}$ & $3.145683 \times 10^{-13041}$ & $3.538653 \times 10^{-6521}$ \\
8 & $0.000000 \times 10^{+00}$ & $0.000000 \times 10^{+00}$ & $2.529895 \times 10^{-52160}$ & $1.003533 \times 10^{-26080}$ \\
9 & $0.000000 \times 10^{+00}$ & $0.000000 \times 10^{+00}$ & $1.058406 \times 10^{-208636}$ & $6.490926 \times 10^{-104319}$ \\
10 & $0.000000 \times 10^{+00}$ & $0.000000 \times 10^{+00}$ & $3.242295 \times 10^{-834542}$ & $1.136074 \times 10^{-417271}$ \\
\hline
\end{tabular}

In this case, (3.44) is written as

$$
\eta \leq \frac{\left(2-\delta_{3}\right) p_{0}}{2}
$$

(2) The ratio of convergence ' $q \eta$ ' given in Proposition 3.3 can be smaller than ' $\sqrt[3]{5} \theta$ ' given in Theorem 2.1 for $q$ close to $\sqrt[3]{b}$ and $M, N, L$ not all zero and $\eta>0$.

Set $\alpha=\sqrt[3]{b} \eta$ and $\beta=\sqrt[3]{5} \theta$. Note that $b<K$ and $40 K^{3}>b$. By comparing $\alpha$ and $\beta$, we have

$$
h_{0}=\frac{1}{2 K}\left(1-\left[\left(\frac{40 K^{3}}{b}\right)^{1 / 6}-1\right]\right) \text {. }
$$

Case 1. If $2.666 M^{3}+0.444 N M-6.740740 L \leq 0$ or $2.666 M^{3}+0.444 N M-6.740740 L>$ 0 and $\eta>h_{0}$, then we have

$$
\alpha<\beta
$$

Case 2. If $2.666 M^{3}+0.444 N M-6.740740 L>0$ and $\eta<h_{0}$, then we have

$$
\alpha>\beta \text {. }
$$

Case 3. If $0<\eta=h_{0}$, then we have

$$
\alpha=\beta \text {. }
$$

Note that the $p$-Jarratt-type method $(p \in[0,1])$ given in [8] uses (2.1)-(2.5), but the sufficient convergence conditions are different from the ones given in the study and guarantees only third-order convergence (not fourth obtained here) in the case of the Jarratt method (for $p=2 / 3$ ).

\section{Conclusions}

We developed a semilocal convergence analysis, using recurrent functions, for the Jarratt method to approximate a locally unique solution of a nonlinear equation in a Banach space. A numerical example and some favorable comparisons with previous works are also reported. 
Competing interests

The authors declare that they have no competing interests.

\section{Authors' contributions}

All authors jointly worked on the results and they read and approved the final manuscript.

\section{Author details}

${ }^{1}$ Department of Mathematical Sciences, Cameron University, Lawton, 73505-6377, Oklahoma. ${ }^{2}$ Department of Mathematics Education and the RINS, Gyeongsang National University, Jinju, 660-701, Korea. ${ }^{3}$ Department of Engineering, Stord Haugesund University College, Stord, Norway.

\section{Acknowledgements}

The second author was supported by the Basic Science Research Program through the National Research Foundation of Korea (NRF) funded by the Ministry of Education, Science and Technology (Grant Number: 2012-0008170).

Received: 29 November 2012 Accepted: 7 April 2013 Published: 22 April 2013

\section{References}

1. Argyros, IK: Convergence and Applications of Newton-Type Iterations. Springer, New York (2008)

2. Argyros, IK: On the Newton-Kantorovich hypothesis for solving equations. J. Comput. Appl. Math. 169, $315-332$ (2004)

3. Argyros, IK: A unifying local-semilocal convergence analysis and applications for two-point Newton-like methods in Banach space. J. Math. Anal. Appl. 298, 374-397 (2004)

4. Argyros, IK, Chen, D, Qian, Q: An inverse-free Jarratt type approximation in a Banach space. Approx. Theory Its Appl. 12, 19-30 (1996)

5. Argyros, IK, Cho, YJ, Hilout, S: Numerical Methods for Equations and Its Applications. CRC Press, New York (2012)

6. Candela, S, Marquina, A: Recurrence relations for rational cubic methods: I. The Halley method. Computing 44, 169-184 (1990)

7. Candela, S, Marquina, A: Recurrence relations for rational cubic methods. II. The Chebyshev method. Computing 45, 355-367 (1990)

8. Ezquerro, JA, Hernández, MA: Avoiding the computation of the second Fréchet-derivative in the convex acceleration of Newton's method. J. Comput. Appl. Math. 96, 1-12 (1998)

9. Jarratt, P: Multipoint iterative methods for solving certain equations. Comput. J. 8, 398-400 (1965/1966)

10. Jarratt, P: Some efficient fourth order multipoint methods for solving equations. Nordisk Tidskr. Informationsbehandling BIT 9, 119-124 (1969)

11. Ostrowski, AM: Solution of Equations in Euclidean and Banach Spaces. Third edition of Solution of equations and systems of equations. Pure and Applied Mathematics, vol. 9. Academic Press, New York (1973)

doi:10.1186/1029-242X-2013-194

Cite this article as: Argyros et al.: On a new semilocal convergence analysis for the Jarratt method. Journal of Inequalities and Applications 2013 2013:194.

\section{Submit your manuscript to a SpringerOpen ${ }^{\circ}$ journal and benefit from:}

- Convenient online submission

Rigorous peer review

- Immediate publication on acceptance

- Open access: articles freely available online

- High visibility within the field

- Retaining the copyright to your article 